Astronomische Zeichen und Abkürzungen

$\begin{gathered}\text { Bezeichnung } \\ \text { der } \\ \text { Wochentage }\end{gathered}$
$\odot$ Sonntag
\& Montag
o Dienstag
† Mittwoch
24 Donnerstag
क Freitag
† Sonnabend

Adspekten

$\sigma$ Konjunktion

$\square$ Quadratur

8 Opposition

Mondphasen

- Neumond

D Erstes Viertel

Vollmond

([ Letztes Vierte]

ภofsteigender

9 Absteigender

Knoten

\title{
Zeichen des Tierkreises und der Himmelskörper
}

\begin{tabular}{|c|c|c|c|c|}
\hline Widder & . & 0 & Grad & \\
\hline ४ Stier & . & 30 & " & $\odot$ Sonne \\
\hline Zwillinge & . & . 60 & $"$ & ( Mond \\
\hline Krebs . . & . & . 90 & $"$ & ఫ Merkur \\
\hline Löwe & . & . 120 & $"$ & 우 Venus \\
\hline Jungfrau. & . & . 150 & $"$ & $\succsim$ Erde \\
\hline Waage & 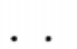 & . 180 & $"$ & $\sigma^{x}$ Mars \\
\hline Skorpion & . & . 210 & $"$ & 4 Jupiter \\
\hline Schütze . & & . 240 & $"$ & $\hbar$ Saturn \\
\hline Steinbock & . . & . 270 & $"$ & $\odot$ Uranus \\
\hline zsserman & in . & . 300 & $n$ & $\Psi$ Neptun \\
\hline ische. & & 330 & » & P Pluto \\
\hline
\end{tabular}

Herausgeber Astronomisches Recheninstitut der Deutschen Akademie der Wissenschaften zu Berlin und Astronomisches Recheninstitut zu Heidelberg Verantwortlich für den Inhalt. Prof Dr A Kahrstedt, Berlin-Lichterfelde-West, únd Prof Dr. W. Fricke, Heidelberg. Verlag. Akademie-Verlag GmbH, Berlin W 8, Mohrenstraße 39; Fernsprecher. Sammelnummer 2003 86; Postschedkkonto: Berlin 35021 Bestell- und Verlagsnummer dieses Werkes: 1004/183. Pre1s 12 DM. Druck: (III/9/1) Såchsische Zeitung. Verlag and Drudkerei, Dresden N 23 9032. Lizenz-Nr 202 - 100/500/56 - MdI der DDR Nr 2561/K 11 